Nr. $251 / 2000$

Some Regularity Results For The Stochastic

Pressure Equation of Wick-Type

Fred Espen Benth and Thomas Gorm Theting 


\title{
SOME REGULARITY RESULTS FOR THE STOCHASTIC PRESSURE EQUATION OF WICK-TYPE
}

\author{
FRED ESPEN BENTH AND THOMAS GORM THETING
}

\begin{abstract}
We prove that the solution of the stochastic pressure equation of Wick-type belongs to a space of generalized random fields having square integrable homogeneous chaos kernels. We find the chaos expansion, and calculate its stochastic regularity in distributional sense. Furthermore, we show that the solution is stable under perturbations of the permeability field and the source term.
\end{abstract}

\section{INTRODUCTION}

We consider the stochastic pressure equation of Wick-type

$$
\begin{aligned}
-\nabla(K(x) \diamond \nabla p(x)) & =f(x) \text { on } \mathcal{D}, \\
p(x) & =0 \text { on } \partial \mathcal{D},
\end{aligned}
$$

where $K$ is a (stochastic) permeability field, $f$ is the source term and $p(x)$ is the unknown pressure. The problem is posed on a bounded domain $\mathcal{D}$ in $\mathbb{R}^{d}$. By introducing the Wick product $\diamond$ in the pressure model we use an Itô interpretation of the stochastic partial differential equation. This product will "mix" the paths in a certain sense, contrary to the ordinary product which would lead to a pathwise ( $\omega$-wise) equation. This model has been proposed and studied intensively by Holden, Øksendal, Ubøe and Zhang [13] as a model for the pressure distribution in a field where the permeability has large fluctuations on all scales (the main example being an oil reservoir). They derive an explicit solution when the permeability is modelled as a lognormal random field. The main objective of this paper is to study (stochastic) regularity properties of their solution together with stability with respect to the data in the problem.

Several authors (see e.g. Holden et al. [13], Benth, Deck and Potthoff [4] and Våge [20]) have used the so-called Kondratiev and Hida spaces of generalized random variables as a convenient setting for studying stochastic (partial) differential equations of Wick-type. Generalized random variables belonging to these distribution spaces may have highly singular chaos kernels as they allow for kernels in the space of tempered Schwartz distributions (see Kondratiev and Streit [15] and Hida, Kuo, Potthoff and Streit [12] for the definition of these spaces). Recently, Grothaus [11] introduced spaces of generalized random variables with chaos kernels having $L^{2}$-regularity, extending work by Potthoff and Timpel [18]. These spaces seem to be sufficiently flexible for the study of a large class of stochastic (partial) differential equations and at the same time providing an improvement of stochastic regularity. Indeed, Grothaus [11] derives explicit solutions for some stochastic (partial) differential equations of Wick-type using these distribution spaces and Wick calculus (see also Kondratiev, Leukert and Streit [14] for Wick calculus).

Date: 21 st February 2000

Key words and phrases. Stochastic partial differential equation, white noise analysis.

The first author acknowledges MaPhySto for financial support. MaPhySto - Centre for Mathematical Physics and Stochastics - is funded by a grant from the Danish National Research Foundation. The second author acknowledges the financial support from the Norwegian Research Council, NFR-grant 131908/410. Discussions with Thomas Deck, Helge Holden and Jürgen Potthoff are greatly appreciated. The second author would like to give special thanks to Jürgen Potthoff and everyone at Lehrstuhl für Mathematik $V$ for making his stay in Mannheim so pleasant. 
From the Wiener-Itô-Segal chaos expansion for random variables with finite variance we know that the chaos kernels belong to the space of square integrable symmetric functions. The generalized random variables of Grothaus have chaos expansions which after truncation belong to the space of square integrable random variables. Also, by a suitable scaling via the Number operator, we get random variables with finite variance. Of course, this offers a significant improvement of the stochastic regularity compared to the Hida and Kondratiev spaces. Besides being interested in more regular solutions of stochastic differential equations, questions like adaptedness and martingale property are central. Having solutions belonging to spaces with $L^{2}$-kernels, these properties are easily verified (see e.g. Benth and Potthoff [7] and Grothaus [11]). See Deck et al. [8] for the generalization to Hida spaces.

We will in this paper study the problem given in (1.1) with a lognormal permeability field in light of the new spaces of Grothaus. Being a problem which intend to model real-world situations, we believe it is of importance to improve the stochastic regularity of the solution. In addition to showing that the solution is much smoother stochastically than proved by Holden et al. [13], we calculate explicitly its chaos expansion. The chaos expansion is a key-point for studying stochastic properties and numerical approximations of the pressure (see e.g . Benth and Gjerde [5] and Theting [19] for numerical studies of the pressure equation). However, further analysis in these directions is outside the scope of the present paper. Another important aspect is stability with respect to data. We shall prove that the pressure depends continuously with respect to the source function $f$ and the covariance structure of the permeablity field. In real applications it is important to know the stability properties of $p(x)$ with respect to its data. The specification of the source or the permeability may only be an approximation to the physical structure, or measurements may be inaccurate. It is therefore of interest to know that small perturbations in the data lead to minor changes in the solution. Our results assure that this is indeed the case for the stochastic pressure.

Modelling with the Wick product is questionable, and need to be justified physically and/or mathematically. As argued in Holden et al. [13], stochastic (partial) differential equations may have singular solutions which make nonlinearities hard to interpret. Understanding products as Wick-products avoid these problems and at the same time provide a nice structure making the equations tractable for Wick calculus techniques. Also, there is a close connection between the Wick product and classical Itô integration (see e.g. Hida et al. [12], Holden et al. [13] and Benth [1]). The Wick product appears naturally in ordinary stochastic differential equations of Itô type via this relation. This indicates that the Wick product performs a similar "mixing" of the paths as does the Itô integral, and one can say that by applying the Wick product in stochastic partial differential equations one get an Itô interpretation of the noise. Whether or not the Wick product gives a good model is still an unanswered question. There are some work done in trying to compare the two ways of interpreting the products, see e.g. [13] (and also the last section in this paper). The results presented here are a step on the way to answer these questions, since we establish regularity of the solution not previously known for our specific Wick-product equation. Furthermore, we shall study a relation between models using the different products which may shed some light on Wick modelling. A pressure equation in one dimension is considered, with combined Dirichlet-Neumann conditions. For this problem we provide a simple relation for the solutions of the two models. We do not go into details about existence and uniqueness of solutions, which can be proven by using techniques from Holden et al. [13]. We also note that the change of boundary condition make the presentation of the connection a great deal simpler. Admittedly, this part is a bit outside the main focus of the paper since we change the formulation of the pressure equation slightly. However, we feel that it provides some understanding of Wick modelling which may be of use for practical understanding of the stochastic pressure equation. We remark in passing that to prove the relation we need some interesting properties of stochastic translation and Wick products which we believe can be useful in other connections.

Here is an outline of the paper: In Section 2 we give the necessary background to white noise analysis. A precise formulation of the stochastic pressure equation of Wick-type together with the 
explicit solution is presented in Section 3. In Section 4 we prove the stochastic regularity of the pressure, following with its Wiener-Itô-Segal chaos expansion in the next section. Stability with respect to data is considered in Section 6. Finally, we state the relation between the Wick model and the model with ordinary product in one dimension when the boundary conditions are slightly changed.

\section{MATHEMATICAL PRELIMinaRies}

White noise analysis is the natural framework for the treatment of the stochastic pressure equation of Wick-type. For a detailed and complete account on the basics of this analysis, we refer the interested reader to the book by Hida, Kuo, Potthoff and Streit [12] (see also Potthoff and Timpel [18], Våge [20], Kuo [16] and Grothaus [11]). This section gives a more or less self-contained presentation of the part of white noise analysis needed for our purposes. We refer to Grothaus [11] for a more complete description of the theory presented below.

We work on the the white noise probability space $\left(S^{\prime}\left(\mathbb{R}^{d}\right), \mathcal{B}\left(\mathcal{S}^{\prime}\left(\mathbb{R}^{d}\right)\right), \mu\right)$. Here $\mathcal{S}^{\prime}\left(\mathbb{R}^{d}\right)$ denotes the space of tempered distributions, i.e. the space of bounded linear functionals on the Schwarz functions on $\mathbb{R}^{d} \cdot \mathcal{B}\left(\mathcal{S}^{\prime}\left(\mathbb{R}^{d}\right)\right)$ denotes the family of Borel subsets of $\mathcal{S}^{\prime}\left(\mathbb{R}^{d}\right)$ generated by the weak-* topology. The characteristic function of the probability measure $\mu$ satisfies

$$
E\left[e^{i\langle\cdot, \phi\rangle}\right]:=\int_{\mathcal{S}^{\prime}} e^{i\langle\omega, \phi\rangle} d \mu(\omega)=e^{-|\phi|_{2}^{2} / 2},
$$

where $|\cdot|_{2}$ is the usual norm in $L^{2}\left(\mathbb{R}^{d}\right)$. The existence of such a $\mu$ is assured by the Bochner-Minlos Theorem.

For $n \in \mathbb{N}_{0}$ (the set of natural numbers including zero), let $(\mathcal{S})^{\widehat{\otimes} n}, \widehat{L_{n}^{2}}$ and $\left(\mathcal{S}^{\prime}\right)^{\widehat{\otimes} n}$ denote the $n$th symmetric tensor products (see e.g. Hida et al. [12]) of $\mathcal{S}\left(\mathbb{R}^{d}\right), L^{2}\left(\mathbb{R}^{d}\right)$ and $\mathcal{S}^{\prime}\left(\mathbb{R}^{d}\right)$, respectively. For notational convenience we put $(\mathcal{S})^{\widehat{\otimes} 0}=\widehat{L_{0}^{2}}=\left(\mathcal{S}^{\prime}\right)^{\widehat{\otimes} 0}=\mathbb{R}$. The norm on $\widehat{L_{n}^{2}}$ is denoted by $|\cdot|_{\widehat{L_{n}^{2}}}$, and simply $|\cdot|_{2}$ when there is no chance of confusion. For each $\phi^{(n)} \in(\mathcal{S})^{\otimes n}$ we define the corresponding smooth Wick monomial

$$
I_{n}\left(\phi^{(n)}\right):=\left\langle: \omega^{\otimes n}:, \phi^{(n)}\right\rangle
$$

where $\mathcal{S}^{\prime} \ni \omega \mapsto: \omega^{\otimes n}: \in\left(S^{\prime}\right)^{\otimes n}$ is the $n$th Wick power of $\omega$ (see [11, 12]). The functions that go inside the Wick monomials are often refered to as the homogeneous chaos kernels. Given two chaos kernels $f^{(n)} \in(\mathcal{S})^{\bar{\otimes} n}$ and $h^{(m)} \in(\mathcal{S})^{\otimes}{ }^{\otimes}$ then

$$
\mathrm{E}\left[I_{n}\left(f^{(n)}\right) I_{m}\left(h^{(m)}\right)\right]=\delta_{n, m} n !\left(f^{(n)}, h^{(n)}\right),
$$

where $\delta_{n, m}$ denotes the Kronecker delta and $(\cdot, \cdot)$ is the usual inner product in $\widehat{L_{n}^{2}}$. We use $\left(L^{p}\right)$ as a short-hand notation for $L^{p}\left(\mathcal{S}^{\prime}\left(\mathbb{R}^{d}\right), \mathcal{B}, \mu\right)$, the space of random variables with finite $p$-moment. The norm in $\left(L^{p}\right)$ will be denoted $\|\cdot\|_{\left(L^{p}\right)}$. It can be shown that given any sequence of smooth kernels $\left\{\phi_{j}^{(n)}\right\}_{j=0}^{\infty}$ converging to $f^{(n)}$ in $\widehat{L_{n}^{2}}$, then the sequence $I_{n}\left(\phi_{j}^{(n)}\right)$ converges to $I_{n}\left(f^{(n)}\right)$ in any $\left(L^{p}\right)$ for $p \geq 1$. It follows that we can define Wick monomials for chaos kernels in $\widehat{L_{n}^{2}}$ and that the orthogonality property (2.1) still holds. For $h \in L^{2}\left(\mathbb{R}^{d}\right)$ we denote the stochastic variable $\omega \mapsto\langle\omega, h\rangle=I_{1}(h)$ by $W_{h}$. This variable is often called smoothed white noise. Let $\mathcal{P}$ denote the space of smooth Wick polynomials on $\mathcal{S}^{\prime}\left(\mathbb{R}^{d}\right)$ :

$$
\mathcal{P}:=\left\{\phi \mid \phi(\omega)=\sum_{n=0}^{N} I_{n}\left(\phi^{(n)}\right)(\omega), \phi^{(n)} \in \mathcal{S}^{\widehat{\otimes} n}, \omega \in \mathcal{S}^{\prime}, N \in \mathbb{N}_{0}\right\}
$$

The space $\mathcal{P}$ is dense in $\left(L^{2}\right)$ (see [12]) and we have the following important result (a proof is given for the one-dimensional case in [12], see also Lemma 4.2 in [17]): 
Theorem 2.1. Every $f \in\left(L^{2}\right)$ has a unique Wiener-Itô-Segal chaos expansion

$$
f(\omega)=\sum_{n=0}^{\infty} I_{n}\left(f^{(n)}\right)(\omega)
$$

with chaos kernels $f^{(n)}$ in $\widehat{L_{n}^{2}}$. Furthermore,

$$
\|f\|_{\left(L^{2}\right)}^{2}=\sum_{n=0}^{\infty} n !\left|f^{(n)}\right|_{2}^{2} .
$$

We proceed to construct our basic pair of smooth and generalized random variables. Our construction follow the ideas in Potthoff and Timpel [18], Grothaus [11] and Benth [3] and others. Let $N$ denote the Ornstein-Uhlenbeck Operator (or Number Operator), defined to act on the $n$th homogeneous chaos of $\left(L^{2}\right)$ as multiplication by $n\left(n \in \mathbb{N}_{0}\right)$. For $b \in[0,1]$ and $q \geq 0$ define the norm

$$
\|\Phi\|_{b, q}=\left\|(N !)^{b / 2} e^{q N} \Phi\right\|_{\left(L^{2}\right)} .
$$

and let $\mathcal{G}_{q}^{b}$ be the completion of $\mathcal{P}$ under this norm. For any $\Phi \in \mathcal{G}_{q}^{b}$ we get

$$
\|\Phi\|_{b, q}^{2}=\left\|\sum_{n=0}^{\infty}(n !)^{b / 2} e^{n q} I_{n}\left(\phi^{(n)}\right)\right\|_{\left(L^{2}\right)}^{2}=\sum_{n=0}^{\infty}(n !)^{1+b} e^{2 n q}\left|\phi^{(n)}\right|_{2}^{2} .
$$

using (2.2), the definition of $N$ and the orthogonality (2.1). The corresponding dual space $\mathcal{G}_{-q}^{-b}$ is characterized as the completion of $\mathcal{P}$ under the norm $\|\cdot\|_{-b,-q}$. We denote the pairing between $\mathcal{G}_{q}^{b}$ and its dual by $\langle\langle\cdot, \cdot\rangle\rangle$ and we have

$$
\langle\langle\Phi, \psi\rangle\rangle=\sum_{n=0}^{\infty} n !\left(\Phi^{(n)}, \psi^{(n)}\right) .
$$

It is clear from the norm expression (2.5) that $\mathcal{G}_{q^{\prime}}^{b} \subset \mathcal{G}_{q}^{b}$ whenever $q^{\prime} \geq q$. Now set

$$
\mathcal{G}^{b}:=\bigcap_{q \geq 0} \mathcal{G}_{q}^{b}
$$

and equip $\mathcal{G}^{b}$ with the projective limit topology. By definition $\mathcal{G}^{-b}$ is the dual of $\mathcal{G}^{b}$ and we have

$$
\mathcal{G}^{-b}=\bigcup_{b \geq 0} \mathcal{G}_{-q}^{-b}
$$

endowed with the inductive limit topology. The pairing between the space of smooth functions $\mathcal{G}^{b}$ and the space of generalized functions $\mathcal{G}^{-b}$ is again given by (2.6). For $b=0$ we denote the corresponding pair of spaces by $\mathcal{G}$ and $\mathcal{G}^{*}$, respectively. This case was studied in Potthoff and Timpel [18] and several important results given here extend to $\mathcal{G}^{b}$ and $\mathcal{G}^{-b}$ for any $b \in[0,1]$. For example it is straightforward to show by the same arguments as in [18] that the space $\mathcal{G}^{b}$ is a reflexive Frechét space for every choice of $b$ in $[0,1]$. See also Benth [3] where a more general setting of weights based on the Number Operator is studied. Let $(\mathcal{S})^{b}$ and $(\mathcal{S})^{-b}$ denote the pair of Kondratiev test- and generalized function spaces (see Kondratiev and Streit [15]). The following theorem establishes relations between these spaces

Theorem 2.2. For each $b \in[0,1]$ the following chain of dense embeddings holds

$$
(\mathcal{S})^{b} \subset \mathcal{G}^{b} \subset\left(L^{2}\right) \subset \mathcal{G}^{-b} \subset(\mathcal{S})^{-b} .
$$

Proof. The proof follows the line of argument of Potthoff and Timpel [18] for the corresponding relation when $b=0$. Note that we only need to show that $(\mathcal{S})^{b} \subset \mathcal{G}^{b}$. The main idea is to consider the norm expression $\|\phi\|_{b, q}=\left\|(N !)^{b / 2} e^{q N} \phi\right\|_{\left(L^{2}\right)}$ together with the properties of the family of norms defining $(\mathcal{S})^{b}$ and use Nelson's hypercontractivity theorem. For more details we refer the reader to $[18]$. 
The following example gives an illustration of an element in our test function spaces

Example 2.1. The Wick exponential is defined by

$$
\exp ^{\circ}\left(W_{\eta}\right):=\frac{\exp \left(W_{\eta}\right)}{E\left[\exp \left(W_{\eta}\right)\right]}=\exp \left(W_{\eta}-\frac{|\eta|_{2}^{2}}{2}\right)
$$

for any $\eta \in \mathcal{S}$ and where $W_{\eta}(\omega)=\langle\omega, \eta\rangle$. The corresponding chaos expansion is

$$
\exp ^{\circ}\left(W_{\eta}\right)=\sum_{n=0}^{\infty} I_{n}\left(\frac{\eta^{\otimes n}}{n !}\right)
$$

and we have

$$
\left\|\exp ^{\circ}\left(W_{\eta}\right)\right\|_{b, q}^{2}=\sum_{n=0}^{\infty}(n !)^{1+b} e^{2 q n}\left|\frac{\eta^{\otimes n}}{n !}\right|_{2}^{2}=\sum_{n=0}^{\infty}(n !)^{-(1-b)} e^{2 q n}|\eta|_{2}^{2 n} .
$$

From this we conclude that if $0 \leq b<1$ then $\exp ^{\diamond}\left(W_{\eta}\right)$ is in $\mathcal{G}_{q}^{b}$ for all $q \geq 0$ (and therefore also in $\left.\mathcal{G}^{b}\right)$. Furthermore, if $b=1$ then $\exp ^{\circ}\left(W_{\eta}\right)$ is in $\mathcal{G}_{q}^{1}$ only as long as $e^{q}|\eta|_{2}<1$.

It is important to notice that the spaces $\mathcal{G}^{ \pm b}$ have elements where the chaos kernels are functions in $\hat{L}_{n}^{2}$. This is not the case for the Kondratiev and Hida spaces, where for instance a Hida distribution in general has chaos belonging to the bigger space of tempered Schwartz distributions. In this sense, $\mathcal{G}^{-b}$ is a much smaller space than $(\mathcal{S})^{-b}$, giving a great deal of extra information on the stochastic regularity. In light of the Wiener-Itô-Segal chaos expansion, elements of $\mathcal{G}^{-b}$ are closer to more familiar (from a probabilistic point of view) square integrable random variables.

We define the $\mathcal{S}$-transform on $\mathcal{G}^{-b}$ analogous to what has been done for the Kondratiev spaces $(\mathcal{S})^{-b}$ (and many other spaces). Note that we have to take special care of the case $b=1$ because of the properties of the Wick exponential (cf. Example 2.1 above).

Definition 2.1. Given $\Phi \in \mathcal{G}^{-b}$ for $0 \leq b<1$. Then the $\mathcal{S}$-transform of $\Phi$ is defined as the map $\mathcal{S} \Phi(\cdot): \mathcal{S}\left(\mathbb{R}^{d}\right) \mapsto \mathbb{R}$ given by

$$
\mathcal{S} \Phi(\eta):=\left\langle\left\langle\Phi, \exp ^{\diamond}\left(W_{\eta}\right)\right\rangle\right\rangle
$$

for all $\eta \in \mathcal{S}\left(\mathbb{R}^{d}\right)$. For every $\Phi \in \mathcal{G}^{-1}$ there is a smallest $q \geq 0$ (called the order of $\Phi$ ) such that $\Phi \in \mathcal{G}_{-q}^{-1}$. For this $q$ we define the $\mathcal{S}$-transform of $\Phi$ as in (2.9), but only for those $\eta$ such that $e^{q}|\eta|_{2}<1$.

Let $\Phi \in \mathcal{G}^{-b}, b \in[0,1]$ and with chaos expansion given by $\Phi=\sum_{n=0}^{\infty} I_{n}\left(\Phi^{(n)}\right)$. Taking the $\mathcal{S}$-transform gives

$$
\mathcal{S} \Phi(\eta)=\left\langle\left\langle\Phi, \exp ^{\diamond}\left(W_{\eta}\right)\right\rangle\right\rangle=\sum_{n=0}^{\infty}\left(\Phi^{(n)}, \eta^{\otimes n}\right)
$$

which is defined for all $\eta \in \mathcal{S}\left(\mathbb{R}^{d}\right)$ if $b \in[0,1)$ and on a suitable neighbourhood of zero if $b=1$. By Cauchy-Schwarz' inequality we get

$$
|\mathcal{S} \Phi(\eta)| \leq \sum_{n=0}^{\infty}\left|\Phi^{(n)}\right|_{2}\left|\frac{\eta^{\otimes n}}{n !}\right|_{2} n !^{(1-b+1+b) / 2} e^{-q n+q n} \leq\|\Phi\|_{-b,-q}\left\|\exp ^{\circ}\left(W_{\eta}\right)\right\|_{b, q}
$$

It follows that $\mathcal{S} \Phi$ is real analytic on $\mathcal{S}\left(\mathbb{R}^{d}\right)$ for all $b \in[0,1)$ and real analytic in a neighborhood of zero for $b=1$ (cf. Example 2.1). Let $\mathcal{S}_{\mathbb{C}}$ denote the complexification of $\mathcal{S}\left(\mathbb{R}^{d}\right)$. The $\mathcal{S}$-transform is a surjective map from $\mathcal{G}^{-b}$ into a subspace of holomorphic functions on $\mathcal{S}_{\mathbb{C}}$ for $b \in[0,1)$, and a surjective map from $\mathcal{G}^{-1}$ into a subspace of functions on $\mathcal{S}_{\mathbb{C}}$ analytic in a neighbourhood of zero. The reader is refered to Grothaus [11] for a complete description of the Characterization Theorem in these cases.

Definition 2.2. Given $\Phi \in \mathcal{G}_{-q_{1}}^{-b_{1}}, \Psi \in \mathcal{G}_{-q_{2}}^{-b_{2}}$. Then their Wick product is defined as the element

$$
\Phi \diamond \Psi:=\mathcal{S}^{-1}(\mathcal{S} \Phi \cdot \mathcal{S} \Psi) \text {. }
$$


It is clear from the definition that the Wick product is associative, commutative and distributive. Furthermore, if $\Phi=\sum_{n=0}^{\infty} I_{n}\left(\Phi^{(n)}\right)$ and $\Psi=\sum_{n=0}^{\infty} I_{n}\left(\Psi^{(n)}\right)$. Then

$$
\Phi \diamond \Psi=\sum_{n=0}^{\infty} I_{n}\left(Z^{(n)}\right) \quad \text { where } \quad Z^{(n)}=\sum_{m=0}^{n} \Phi^{(n-m)} \widehat{\otimes} \Psi^{(m)}
$$

by uniquness of the $\mathcal{S}$-transform. The next proposition and its corollary establish a regularity property of the Wick product.

Proposition 2.3. Given $\Phi \in \mathcal{G}_{-q_{1}}^{-b_{1}}, \Psi \in \mathcal{G}_{-q_{2}}^{-b_{2}}$ for $b_{i} \in[0,1], q_{i} \geq 0, i=1,2$. Let $b=\max \left(b_{1}, b_{2}\right)$ and set $q_{0}=\ln (2)(1-b) / 2+\max \left(q_{1}, q_{2}\right)$. Then $\Phi \diamond \Psi \in \mathcal{G}_{-q}^{-b}$ for any $q>q_{0}$ and

$$
\|\Phi \diamond \Psi\|_{-b,-q} \leq\left(1-e^{-\left(q-q_{0}\right)}\right)^{-1}\|\Phi\|_{-b_{1},-q_{1}}\|\Psi\|_{-b_{2},-q_{2}} .
$$

By slightly adjusting two estimates in the proof of Proposition 2.3 given below we can derive the corollary

Corollary 2.4. Given $\Phi \in \mathcal{G}_{q_{1}}^{b_{1}}, \Psi \in \mathcal{G}_{q_{2}}^{b_{2}}$ for $b_{i} \in[0,1], q_{i} \geq 0, i=1,2$. Let $b=\min \left(b_{1}, b_{2}\right)$ and set $q_{0}=\min \left(q_{1}, q_{2}\right)-\ln (2)(1+b) / 2$. Then if $q_{0}>0$ we have $\Phi \diamond \Psi \in \mathcal{G}_{q}^{b}$ for any $0 \leq q<q_{0}$ and

$$
\|\Phi \diamond \Psi\|_{b, q} \leq K_{q}\|\Phi\|_{b_{1}, q_{1}}\|\Psi\|_{b_{2}, q_{2}}
$$

where $K_{q}=\left(1-e^{-\left(q_{0}-q\right)}\right)^{-1}$. If $q_{0} \leq 0$ the result holds for $q=0$ and $K_{q}=\left(1-e^{-\min \left(q_{1}, q_{2}\right)}\right)^{-1}$.

Proof of Proposition 2.3. Take the $\|\cdot\|_{-b,-q}$-norm of the $n$th term in the chaos expansion, recalling the expression for the Wick product in (2.11). Using the triangle inequality and Cauchy-Schwarz' inequality we get

$$
\begin{aligned}
\left\|I_{n}\left(Z^{(n)}\right)\right\|_{-b,-q} & \leq \sum_{m=0}^{n}\left\|I_{n}\left(\Phi^{(n-m)} \widehat{\otimes} \Psi^{(m)}\right)\right\|_{-b,-q} \\
& \leq \sum_{m=0}^{n}(n !)^{(1-b) / 2} e^{-q n}\left|\Phi^{(n-m)}\right|_{2}\left|\Psi^{(m)}\right|_{2} \\
& \leq \sum_{m=0}^{n}\left(\begin{array}{c}
n \\
m
\end{array}\right)^{(1-b) / 2} e^{-q n}(n-m) !^{(1-b) / 2}\left|\Phi^{(n-m)}\right|_{2} m !^{(1-b) / 2}\left|\Psi^{(m)}\right|_{2} .
\end{aligned}
$$

By assumption we have $0 \leq(1-b) \leq\left(1-b_{i}\right)$ for $i=1,2$. Therefore

$$
\begin{aligned}
\left\|I_{n}\left(Z^{(n)}\right)\right\|_{-b,-q} & \leq \sum_{m=0}^{n}\left(\begin{array}{c}
n \\
m
\end{array}\right)^{(1-b) / 2} e^{-q n+q_{1}(n-m)+q_{2} m}\left\|I_{n-m}\left(\Phi^{(n-m)}\right)\right\|_{-b_{1},-q_{1}}\left\|I_{m}\left(\Psi^{(m)}\right)\right\|_{-b_{2},-q_{2}} \\
& \leq e^{-\left(q-q_{0}\right) n} \sum_{m=0}^{n}\left\|I_{n-m}\left(\Phi^{(n-m)}\right)\right\|_{-b_{1},-q_{1}}\left\|I_{m}\left(\Psi^{(m)}\right)\right\|_{-b_{2},-q_{2}} \\
& \leq e^{-\left(q-q_{0}\right) n}\|\Phi\|_{-b_{1},-q_{1}}\|\Psi\|_{-b_{2},-q_{2}}
\end{aligned}
$$

where we used $\left(\begin{array}{l}n \\ m\end{array}\right) \leq 2^{n}$ and Cauchy-Schwarz again. Summing over all $n \in \mathbb{N}_{0}$ proves the result.

From Proposition 2.3 we have

Theorem 2.5. Let $b \in[0,1]$. Then $\mathcal{G}^{b}$ and $\mathcal{G}^{-b}$ are algebras under the Wick product. Moreover, the Wick product is continuous from $\mathcal{G}^{b} \times \mathcal{G}^{b}$ (equipped with the product topology) onto $\mathcal{G}^{b}$.

Now we consider the regularity of the ordinary product.

Proposition 2.6. Given $\phi \in \mathcal{G}_{q_{1}}^{b_{1}}, \psi \in \mathcal{G}_{q_{2}}^{b_{2}}$ where $b_{i} \in[0,1]$ and $q_{i} \geq 0$. Let $b=\min \left(b_{1}, b_{2}\right)$ and set $q_{0}=\min \left(q_{1}, q_{2}\right)-\left[(1+b) b \ln (2)+(1-b) \ln \left(2^{b+1}+1\right)\right] / 2$. If $q_{0}>0$ then $\phi \cdot \psi \in \mathcal{G}_{q}^{b}$ and there is a constant $K_{q}$ depending on $q$ such that

$$
\|\phi \cdot \psi\|_{b, q} \leq K_{q}\|\phi\|_{b_{1}, q_{1}}\|\psi\|_{b_{2}, q_{2}}
$$

for all $0 \leq q<q_{0}$. If $q_{0} \leq 0$ the same result holds with $q=0$ and $K_{q}=1$. 
Proof. The proof is similar to the one given in Potthoff and Timpel [18] for the case $b=0$. We have (see e.g. Hida et al. [12]) that

$$
I_{n}\left(\Phi^{(n)}\right) I_{m}\left(\Psi^{(m)}\right)=\sum_{k=0}^{m \wedge n} k !\left(\begin{array}{c}
m \\
k
\end{array}\right)\left(\begin{array}{l}
n \\
k
\end{array}\right) I_{m+n-2 k}\left(\Phi^{(n)}\right) \widehat{\otimes}_{k} I_{m}\left(\Psi^{(m)}\right)
$$

where $m \wedge n$ denotes $\min (m, n)$. Here

$$
\Phi^{(n)} \otimes_{k} I_{m}\left(\Psi^{(m)}=\int \Phi^{(n)}\left(\cdot, s_{1}, \ldots, s_{k}\right) \Psi\left(\cdot, s_{1}, \ldots, s_{k}\right) d^{k} s \quad(k \leq m \wedge n)\right.
$$

and $\left.\Phi^{(n)}\right) \widehat{\otimes}_{k} I_{m}\left(\Psi^{(m)}\right)$ denotes the corresponding symmetrization. Take the $\|\cdot\|_{b, q}$-norm in (2.12) and use the triangle inequality together with Cauchy-Schwarz to have

$$
\begin{gathered}
\left.\left\|I_{n}\left(\Phi^{(n)}\right) I_{m}\left(\Psi^{(m)}\right)\right\|_{b, q} \leq \sum_{k=0}^{m \wedge n} k !\left(\begin{array}{c}
m \\
k
\end{array}\right)\left(\begin{array}{l}
n \\
k
\end{array}\right)(m+n-2 k) !^{(1+b) / 2} e^{q(m+n-2 k)} \mid \Phi^{(n)}\right)\left.\hat{\otimes}_{k} \Psi^{(m)}\right|_{2} \\
\leq\left\|I_{n}\left(\Phi^{n)}\right)\right\|_{b_{1}, q_{1}} e^{-\left(q_{1}-q\right) n}\left\|I_{m}\left(\Psi^{(m)}\right)\right\|_{b_{2}, q_{2}} e^{-\left(q_{2}-q\right) n}\left(\sum_{k=0}^{m \wedge n}\left[\left(\begin{array}{c}
m \\
k
\end{array}\right)\left(\begin{array}{l}
n \\
k
\end{array}\right)\right]^{(1-b) / 2}\right. \\
\left.\left(\begin{array}{c}
m+n-2 k \\
m-k
\end{array}\right)^{(1+b) / 2} k !^{-b} e^{-2 q k}\right) .
\end{gathered}
$$

The last sum is bounded by

$$
\begin{aligned}
& 2^{(m+n)(1+b) / 2} \sum_{k=0}^{m+n}\left(\begin{array}{c}
m+n \\
2 k
\end{array}\right)^{(1-b) / 2} 2^{-(1+b) k} \\
& \leq 2^{(m+n)(1+b) / 2}\left(\sum_{k=0}^{m+n} 2^{-(1+b) k}\right)^{(1+b) / 2}\left(\sum_{k=0}^{m+n}\left(\begin{array}{c}
m+n \\
2 k
\end{array}\right) 2^{-(1+b) k}\right)^{(1-b) / 2} \\
& \leq K \alpha^{m+n}
\end{aligned}
$$

where $\alpha:=2^{(1+b) / 2}\left(1+2^{-(1+b)}\right)^{(1-b) / 2}$ and $K:=\left(1-2^{-(1+b)}\right)^{-(1+b) / 2}$. It follows that

$$
\left\|I_{n}\left(\Phi^{(n)}\right) I_{m}\left(\Psi^{(m)}\right)\right\|_{b, q} \leq K\left\|I_{n}\left(\Phi^{n)}\right)\right\|_{b_{1}, q_{1}} \sigma^{n}\left\|I_{m}\left(\Psi^{(m)}\right)\right\|_{b_{2}, q_{2}} \sigma^{m}
$$

where $\sigma:=\alpha e^{-\left(\min \left(q_{1}, q_{2}\right)-q\right)}$. Summing over all $m, n \in \mathbb{N}_{0}$ and applying Cauchy-Schwarz twice give

$$
\begin{aligned}
\|\Phi \cdot \Psi\|_{b, q} & \leq K\left(\sum_{m=0}^{\infty}\left\|I_{n}\left(\Phi^{n)}\right)\right\|_{b_{1}, q_{1}} \sigma^{n}\right)\left(\sum_{m=0}^{\infty}\left\|I_{m}\left(\Psi^{(m)}\right)\right\|_{b_{2}, q_{2}} \sigma^{m}\right) \\
& \leq K \frac{1}{1-\sigma^{2}}\|\Phi\|_{b_{1}, q_{1}}\|\Psi\|_{b_{2}, q_{2}} .
\end{aligned}
$$

Note that $\sigma<1$ if and only if $q<\min \left(q_{1}, q_{2}\right)-\ln \alpha$ which explain the assumption on $q$. The last result for $q_{0} \leq 0$ is obvious.

From Proposition 2.6 it follows that

Theorem 2.7. Let $b \in[0,1]$. Then $\mathcal{G}^{b}$ is an algebra under pointwise product. Moreover, the pointwise product is continuous from $\mathcal{G}^{b} \times \mathcal{G}^{b}$ (with product topology) onto $\mathcal{G}^{b}$.

We define in the usual manner (Pettis) integration of parametrized elements of $\mathcal{G}^{-b}$ :

Definition 2.3. Let $(X, \mathcal{M}, d x)$ be a given measure space and let $b \in[0,1]$. A function $\Phi$ : $X \mapsto \mathcal{G}^{-b}$ is said to be $\mathcal{G}^{-b}$ (Pettis) integrable if $\langle\langle\Phi(x), \psi\rangle\rangle$ is in $L^{1}(X, d x)$ for all $\psi$ in $\mathcal{G}^{b}$. The $\mathcal{G}^{-b}$-valued integral of $\Phi(x)$ over any measurable set $E \in \mathcal{M}$ is defined as the (unique) element $\int_{E} \Phi(x) d x$ in $\mathcal{G}^{-b}$ that satisfies

for all $\psi$ in $\mathcal{G}^{b}$.

$$
\left\langle\left\langle\int_{E} \Phi(x) d x, \psi\right\rangle\right\rangle=\int_{E}\langle\langle\Phi(x), \psi\rangle\rangle d x
$$


Remark. It is a consequence of the same argument as for Proposition 8.1 in Hida et al. [12] that $\int_{E} \Phi(x) d x$ exists as an element in $\mathcal{G}^{-b}$. Furthermore, note that from our definition it follows that

$$
\mathcal{S}\left(\int_{E} \Phi(x) d x\right)(\eta)=\int_{E} \mathcal{S} \Phi(x, \eta) d x .
$$

For any $q \geq 0$ and $\phi \in \mathcal{G}^{b}$ we have

$$
\int_{E}|\langle\langle\Phi(x), \phi\rangle\rangle| d x \leq \int_{E}\|\Phi(x)\|_{-b,-q}\|\phi\|_{b, q} d x .
$$

Thus, if $\Phi(x)$ is Bochner integrable on $\mathcal{G}_{-q}^{-b}$ from some $q \geq 0$ then it will be Pettis integrable and the two integrals coincide. We refer the reader to Hida et al. [12] and Yosida [21] for more theory on Pettis and Bochner integration.

\section{The Stochastic PRESSURE Equation}

We formulate the stochastic pressure equation of Wick-type. The problem consists of finding the pressure $p(x)$ on a bounded domain $\mathcal{D}$ (being the 'reservoir') in $\mathbb{R}^{d}$ for $d \in \mathbb{N}$, given a permeability field and source. The pressure is given as the solution to the stochastic partial differential equation,

$$
\begin{aligned}
-\nabla\left(\exp ^{\diamond}\left(W_{\phi}(x)\right) \diamond \nabla p(x)\right) & =f(x) \quad x \text { on } \mathcal{D}, \\
p(x) & =0 \text { on } \partial \mathcal{D},
\end{aligned}
$$

where $W_{\phi}(x, \omega)=\left\langle\omega, \phi_{x}\right\rangle, \phi_{x}(y)=\phi(y-x), \phi \in \mathcal{S}\left(\mathbb{R}^{d}\right)$, and $\exp ^{\circ}\left(W_{\phi}\right)$. denotes the Wick exponential. $\nabla$ is the gradient with respect to $x$. The permeability field is modelled as $\exp ^{\circ} W_{\phi}(x)$, and is the noise in the system making the pressure stochastic. The noise is smooth due to the smearing effect of $W_{\phi}(x)$. A singular (or white) noise is obtained with the choice $\phi=\delta_{0}$, where $\delta_{0}$ is the Dirac- $\delta$ function at zero. We shall not consider this kind of noise here, but refer to Holden et al. [13] for further details. Furthermore, $f$ is the source term, which in an oil reservoir context models the extraction of oil (and injection of water/gas). To emphasis the dependency on the smearing function $\phi$, we will denote the solution of the pressure equation (3.1) by $p_{\phi}(x)$.

Remark. The map $\mathcal{D} \ni x \mapsto \exp ^{\circ}\left(W_{\phi}\right)(x, \omega)$ defines a random field which is lognormally distributed at every location $x$ in the domain $\mathcal{D}$ since $\left\langle\omega, \phi_{x}\right\rangle$ by definition is a Gaussian field. The covariance structure of the Gaussian random field $W_{\phi}(x, \omega)$ is given by

$$
\operatorname{Cov}\left(W_{\phi}(x), W_{\phi}(y)\right)=\mathrm{E}\left[W_{\phi}(x) W_{\phi}(y)\right]=(\phi * \phi)(y-x)
$$

where $*$ is the convolution product for functions on $\mathbb{R}^{d}$. Note that this gives flexibility of modelling different covariance fields. A major drawback, however, is that $\mathrm{E}\left[\exp ^{\diamond} W_{\phi}(x)\right]=1$, which is independent of $x$. This means that $\exp ^{\curvearrowright}\left(W_{\phi}\right)(x)$ is hardly suitable for modelling permeability variations over larger fields, where there are no reason to expect that the mean of the fluctuations are independent of location.

One reason for introducing the Wick product between the permeability field and the gradient of the pressure in the formulation of (3.1) is due to the fact that solutions are singular (or, in other words, stochastic distributions). This makes the ordinary product hard to interpret, and one way to resolve this is to introduce the Wick product. If the permeability is deterministic, the Wick product will coincide with the ordinary, pointwise product. In this respect, the Wick-type stochastic pressure equation is a generalization of the determinsitic problem. The reader is refered to Holden et al. [13] for further discussions of the modelling aspects using the Wick product.

The map $p_{\phi}: \mathcal{D} \mapsto \mathcal{G}^{-1}$ is said-to be a (weak) solution to (3.1) if

$$
\left\langle\left\langle-\nabla\left(\exp ^{\circ}\left(W_{\phi}(x)\right) \diamond \nabla p_{\phi}(x)\right), \psi\right\rangle\right\rangle=\langle\langle f(x), \psi\rangle\rangle
$$

for every $\psi$ in $\mathcal{G}^{1}$ and every $x$ in $\mathcal{D}$. 
The unique solution of (3.1) is calculated explicitly in Holden et al. [13, Th. 4.6.1] by using $\mathcal{S}$-transformation techniques. It has the form

$$
\begin{aligned}
p_{\phi}(x)= & \frac{1}{2} \exp ^{\circ}\left(-\frac{1}{2} W_{\phi}(x)\right) \diamond \hat{\mathrm{E}}^{x}\left[\int_{0}^{\tau_{\mathcal{D}}} f\left(b_{t}\right) \diamond\right. \\
& \left.\exp ^{\circ}\left[-\frac{1}{2} W_{\phi}\left(b_{t}\right)-\frac{1}{4} \int_{0}^{t}\left(\frac{1}{2}\left(\nabla W_{\phi}(y)\right)^{\diamond 2}+\Delta W_{\phi}(y)\right)_{y=b_{s}} d s\right] d t\right]
\end{aligned}
$$

for $\phi \in \mathcal{S}\left(\mathbb{R}^{d}\right)$ and $x \in \mathcal{D}$. Here $\left(b_{t}\left(\omega_{2}\right), \widehat{P}_{x}\right)$ is a standard Brownian motion on $\mathbb{R}^{d}$ starting in $x$, $\hat{E}^{x}$ is expectation with respect to the probability measure $\widehat{P}_{x}$ on a measure space $(\hat{\Omega}, \hat{\mathcal{F}})$, and we define

$$
\tau_{\mathcal{D}}:=\inf \left\{t \geq 0 \mid b_{t} \notin \mathcal{D}\right\}
$$

That is, $\tau_{\mathcal{D}}$ is the first exit time out of the domain $\mathcal{D}$ for the Brownian motion $b_{t}$ starting in $x$ which is finite $\hat{P}_{x}$-almost surely since $\mathcal{D}$ is bounded. Note that since $\mathcal{D}$ is bounded there exists a positive $\rho$ such that $\hat{\mathrm{E}}^{x}\left[e^{\rho \tau_{\mathcal{D}}}\right]$ is uniformly bounded for all $x \in \mathcal{D}$. In fact, a straighforward calculation shows that it is sufficient to choose $0<\rho<-d \ln P(|X| \leq \operatorname{diam}(\mathcal{D})$ ) where $X$ is a standard Gaussian distributed random variable [9, p. 40].

Before we proceed we need the following definition: Given $b \in[-1.1]$ and $q \in \mathbb{R}$, then define the norm

$$
\|F\|_{b, q, \infty}=\sup _{x \in \mathcal{D}}\|F(x)\|_{b, q}
$$

for functions $F: \mathcal{D} \mapsto \mathcal{G}_{q}^{b}$.

\section{Stochastic Regularity of the PRESSURE}

We prove under natural smoothness conditions for the force term $f$ that $p_{\phi}(x)$ belongs to $\mathcal{G}_{-q}^{-1}$, where we have a lower bound on $q$. The precise result is as follows:

Theorem 4.1. Assume $f(x) \in \mathcal{G}_{-q_{f}}^{-1}$ for all $x \in \mathcal{D}$ and $\|f\|_{-1,-q_{f}, \infty}<\infty$. Let $q \in \mathbb{R}$ be such that $q>\max \left(q_{f}, q_{\rho}\right)$ where $q_{\rho}$ satisfies the inequality

$$
e^{-2 q_{\rho}} \frac{|\nabla \phi|_{2}^{2}}{8}+e^{-q_{\rho}} \frac{|\Delta \phi|_{2}}{4}<\rho
$$

and where $\rho>0$ is such that $\hat{E}^{x}\left[e^{\rho \mathcal{T}_{\mathcal{D}}}\right]$ is uniformly bounded for $x \in \mathcal{D}$. Then $p_{\phi}(x) \in \mathcal{G}_{-q}^{-1}$ for all $x \in \mathcal{D}$ and $\left\|p_{\phi}\right\|_{-1,-q, \infty}<\infty$.

Before we give the proof of Theorem 4.1, we state the following useful result

Lemma 4.2. Let $t \mapsto \phi_{t} \in \widehat{L_{n}^{2}}$ be given and continuous in $t$. Then for any interval $[a, b] \subset \mathbb{R}$ we have

$$
\int_{a}^{b} I_{n}\left(\phi_{t}\right) d t=I_{n}\left(\int_{a}^{b} \phi_{t} d t\right)
$$

Proof. Integrability of $I_{n}\left(\phi_{t}\right)$ follows from the continuity of $\phi_{t}$. Taking the $\mathcal{S}$-transform of the left-hand side and using (2.13) gives

$$
\mathcal{S}\left(\int_{a}^{b} I_{n}\left(\bar{\phi}_{t}\right) d t\right)(\eta)=\int_{a}^{b}\left(\phi_{t}, \eta^{\widehat{\otimes} n}\right) d t=\left(\int_{a}^{b} \phi_{t} d t, \eta^{\widehat{\otimes} n}\right)
$$

where the last equality follows using Fubini's theorem. The Lemma now follows by uniqueness of the $\mathcal{S}$-transform. 
Proof of Theorem 4.1. Let $q_{1}$ be such that $q>q_{1}>\max \left(q_{f}, q_{\rho}\right)$. By Proposition 2.3 with $b=1$ we have,

$$
\left\|p_{\phi}(x)\right\|_{-1,-q} \leq C_{1}\left\|\exp ^{\circ}\left(-\frac{1}{2} W_{\phi}(x)\right)\right\|_{-1,0} \cdot\left\|\hat{\mathrm{E}}^{x}\left[\int_{0}^{\tau_{\mathcal{D}}} \cdots d t\right]\right\|_{-1,-q_{1}}
$$

where $C_{1}=\left(1-e^{-\left(q-q_{1}\right)}\right)^{-1}$. By Example 2.1 and since $\left|\phi_{x}\right|_{2}=|\phi|_{2}$ the first norm on the right hand side in (4.2) is uniformly bounded by $\exp \left(|\phi|_{2} / 2\right)$. We proceed to estimate the last norm. This estimation will at the same time prove Bochner integrability of the integrand. First recall that smoothed white noise has the chaos expansion

$$
W_{\phi}(x)=\left\langle\omega, \phi_{x}\right\rangle=I_{1}\left(\phi_{x}\right) .
$$

By the definition of the wick product we get $\left(D_{i} W_{\phi}\right)^{\diamond 2}(x)=I_{2}\left(\left(D_{i} \phi_{x}\right)^{\otimes 2}\right)$ and hence

$$
\left(\nabla W_{\phi}\right)^{\diamond 2}(x)=I_{2}\left(\left(\nabla \phi_{x}\right)^{\widehat{\otimes} 2}\right) .
$$

Furthermore, we have

$$
\Delta W_{\phi}=\sum_{i=1}^{d} D_{i}^{2} W_{\phi}(x)=I_{1}\left(\Delta \phi_{x}\right)
$$

Recall the exponent in (3.2)

$$
-\frac{1}{2} W_{\phi}\left(b_{t}\right)-\frac{1}{4} \int_{0}^{t}\left(\frac{1}{2}\left(\nabla W_{\phi}(y)\right)^{\diamond 2}+\Delta W_{\phi}(y)\right)_{y=b_{s}} d s .
$$

Define

$$
\begin{aligned}
& \phi_{t}^{(1)}(y)=\frac{1}{2} \phi\left(y-b_{t}\right)+\frac{1}{4} \int_{0}^{t} \Delta \phi\left(y-b_{s}\right) d s \\
& \phi_{t}^{(2)}\left(y_{1}, y_{2}\right)=\frac{1}{8} \int_{0}^{t} \nabla \phi\left(\cdot-b_{s}\right) \widehat{\otimes} \nabla \phi\left(\cdot-b_{s}\right)\left(y_{1}, y_{2}\right) d s .
\end{aligned}
$$

Substituting for (4.3),(4.4) and (4.5) in (4.6) gives

$$
\begin{aligned}
& -\frac{1}{2} I_{1}\left(\phi_{b_{t}}\right)-\frac{1}{4} \int_{0}^{t}\left(\frac{1}{2} I_{2}\left(\left(\nabla \phi_{y}\right)^{\otimes 2}\right)+I_{1}\left(\Delta \phi_{y}\right)\right)_{y=b_{s}} d s \\
& =I_{1}\left(-\frac{1}{2} \phi_{b_{t}}-\frac{1}{4} \int_{0}^{t}\left(\Delta \phi_{y}\right)_{y=b_{s}} d s\right)+I_{2}\left(-\frac{1}{8} \int_{0}^{t}\left(\left(\nabla \phi_{y}\right)^{\otimes 2}\right)_{y=b_{*}} d s\right) .
\end{aligned}
$$

where the last equality follows from Lemma 4.2. Hence, we can write (4.6) as

$$
-I_{1}\left(\phi_{t}^{(1)}\right)-I_{2}\left(\phi_{t}^{(2)}\right)
$$

with $\phi_{t}^{(1)}$ and $\phi_{t}^{(2)}$ given in (4.7). Now we estimate the last norm in (4.2). Choose $q_{2}$ such that $q_{1}>q_{2}>\max \left(q_{f}, q_{\rho}\right)$. Then again using Proposition 2.3 we get

$$
\begin{aligned}
\| \hat{\mathrm{E}}^{x} & {\left[\int_{0}^{\mathcal{T}_{\mathcal{D}}} f\left(b_{t}\right) \diamond \exp ^{\diamond}\left(-I_{2}\left(\phi_{t}^{(2)}\right)-I_{1}\left(\phi_{t}^{(1)}\right)\right) d t\right] \|_{-1,-q_{1}} } \\
& \leq \hat{\mathrm{E}}^{x}\left[\int_{0}^{\tau_{\mathcal{D}}}\left\|f\left(b_{t}\right) \diamond \exp ^{\diamond}\left(-I_{2}\left(\phi_{t}^{(2)}\right)-I_{1}\left(\phi_{t}^{(1)}\right)\right)\right\|_{-1,-q_{1}} d t\right] \\
& \leq \hat{\mathrm{E}}^{x}\left[\int_{0}^{\tau_{\mathcal{D}}} C_{2}\left\|f\left(b_{t}\right)\right\|_{-1,-q_{f}} \cdot\left\|\exp ^{\diamond}\left(-I_{2}\left(\phi_{t}^{(2)}\right)-I_{1}\left(\phi_{t}^{(1)}\right)\right)\right\|_{-1,-q_{2}} d t\right] \\
& \leq C_{3} \hat{\mathrm{E}}^{x}\left[\int_{0}^{\tau_{\mathcal{D}}}\left\|\exp ^{\diamond}\left(-I_{1}\left(\phi_{t}^{(1)}\right)\right)\right\|_{-1,-q_{\rho}}\left\|\exp ^{\diamond}\left(-I_{2}\left(\phi_{t}^{(2)}\right)\right)\right\|_{-1,-q_{\rho}} d t\right] .
\end{aligned}
$$

Here $C_{2}=\left(1-e^{-\left(q_{1}-q_{2}\right)}\right)^{-1}$ and $C_{3}=C_{2}\left(1-e^{-\left(q_{2}-q_{\rho}\right)}\right)^{-1}\|f(x)\|_{-1,-q_{f}, \infty}<\infty$ by assumption. Consider the last norm: By the triangle inequality we have

$$
\left\|\exp ^{\diamond}\left(-I_{2}\left(\phi_{t}^{(2)}\right)\right)\right\|_{-1,-q_{\rho}}=\left\|\sum_{n=0}^{\infty} \frac{(-1)^{n}}{n !} I_{2 n}\left(\phi_{t}^{(2) \otimes}\right)\right\|_{-1,-q_{\rho}}
$$


Cauchy-Schwarz' inequality now gives,

$$
\begin{aligned}
& \leq \sum_{n=0}^{\infty} \frac{1}{n !}\left\|I_{2 n}\left(\phi_{t}^{(2) \otimes \widehat{~} n}\right)\right\|_{-1,-q_{\rho}} \\
& =\sum_{n=0}^{\infty} \frac{1}{n !} e^{-2 n q_{\rho}}\left|\phi_{t}^{(2)}\right|_{2}^{n} .
\end{aligned}
$$

$$
\left|\phi_{t}^{(2)}\right|_{2} \leq \frac{|\nabla \phi|_{2}^{2}}{8} t
$$

and hence,

$$
\left\|\exp ^{\circ}\left(-I_{2}\left(\phi_{t}^{(2)}\right)\right)\right\|_{-1,-q_{\rho}} \leq \sum_{n=0}^{\infty} \frac{1}{n !}\left(e^{-2 q_{\rho}} \frac{|\nabla \phi|_{2}^{2}}{8}\right)^{n} t^{n}=\exp \left(e^{-2 q_{\rho}} \frac{|\nabla \phi|_{2}^{2}}{8} t\right) .
$$

The other norm is equivalently estimated by the triangle inequality

$$
\left\|\exp ^{\circ}\left(-I_{1}\left(\phi_{t}^{(1)}\right)\right)\right\|_{-1,-q_{\rho}} \leq \exp \left(e^{-q_{\rho}}\left(\frac{1}{2}|\phi|_{2}+\frac{1}{4}|\triangle \phi|_{2} t\right)\right)
$$

since by the Cauchy-Schwarz inequality,

$$
\left|\phi_{t}^{(1)}\right|_{2} \leq \frac{1}{2}|\phi|_{2}+\frac{1}{4}|\triangle \phi|_{2} t .
$$

Thus, by (4.2) we get the following bound

$$
\left\|p_{\phi}(x)\right\|_{-1,-q_{\rho}} \leq C_{4} \hat{\mathrm{E}}^{x}\left[\int_{0}^{\tau_{\mathcal{D}}} \exp \left(e^{-2 q_{\rho}} \frac{|\nabla \phi|_{2}^{2}}{8} t+e^{-q_{\rho}} \frac{1}{4}|\triangle \phi|_{2} t\right) d t\right]
$$

where the constant $C_{4}=C_{1} C_{3} \exp \left(\left(1+e^{-q_{p}} / 2\right)|\phi|_{2}\right)$ is independent of $x$. By the assumption on $q_{\rho}$ the theorem is proved.

Recall that elements of $\mathcal{G}_{-q}^{-1}$ have chaos kernels which are square integrable functions. In fact, we may rescale the $n$th chaos function by $(n !)^{-1 / 2} \exp (-q n)$ (for every $n \in \mathbb{N}_{0}$ ) to obtain an element of $\left(L^{2}\right)$. Such a regularization corresponds to applying $(N !)^{-1 / 2} \exp (-q N)$, where $N$ is the Number Operator, to the element of $\mathcal{G}_{-q}^{-1}$. Thus we see that $p_{\phi}(x)$ has square integrable chaos kernels and that a renormalization via the Number Operator gives a random field in $\left(L^{2}\right)$, i.e. $(N !)^{-1 / 2} \exp (-q N) p_{\phi}(x) \in\left(L^{2}\right)$ for all $x \in \mathcal{D}$. In the next section we will derive the chaos expansion of $p_{\phi}(x)$.

\section{ChaOs EXPANSion of the PRESSURE}

In this section we find the chaos expansion of the pressure $p_{\phi}(x)$ solving equation (3.2). This expansion will of course depend on the chaos expansion of the source function $f$, and we shall assume here that this is known. The derivation of the expansion will make use of the $\mathcal{S}$-transform.

Theorem 5.1. Suppose the assumptions of Theorem 4.1 holds, and let $p_{\phi}$ be the solution of the pressure equation given in (3.2). Then the chaos expansion of $p_{\phi}$ is given as

$$
p_{\phi}(x)=\sum_{n=0}^{\infty} I_{n}\left(p_{\phi}^{(n)}(x)\right)
$$

where

$$
\begin{aligned}
p_{\phi}^{(n)}\left(x ; y_{1}, \cdots, y_{n}\right) & =\sum_{l=0}^{n} \sum_{k=0}^{l} \sum_{m=0}^{\lfloor k / 2\rfloor} \frac{(-1)^{n-l}}{2^{n-l+1}(n-l) ! m !(k-2 m) !}\left(\phi_{x}^{\widehat{\otimes} n-l} \widehat{\otimes}\right. \\
& \left.-\hat{E}^{x}\left[\int_{0}^{\tau_{\mathcal{D}}} f_{b_{t}}^{(l-k)} \widehat{\otimes} \eta_{1}^{\widehat{\otimes} k-2 m}(t) \widehat{\otimes} \eta_{2}^{\widehat{\otimes} m}(t) d t\right]\right)\left(y_{1}, \cdots, y_{n}\right)
\end{aligned}
$$

and where $\eta_{1}\left(t ; y_{1}\right):=-\phi_{t}^{(1)}\left(y_{1}\right), \eta_{2}\left(t ; y_{1}, y_{2}\right):=-\phi_{t}^{(2)}\left(y_{1}, y_{2}\right)$. for the functions $\phi^{(1)}$ and $\phi^{(2)}$ defined in (4.7). 
Proof of Theorem 5.2. The idea of the proof is to $\mathcal{S}$-transform the solution in (3.2), reformulate this expression and apply the uniqueness of the $\mathcal{S}$-transform. The details follow: Let $\xi$ be in $\mathcal{S}\left(\mathbb{R}^{d}\right)$, then the $\mathcal{S}$-transform of $p_{\phi}$ in (3.2) evaluated in $\xi$ can be written as

$$
\begin{aligned}
\mathcal{S} p_{\phi}(\xi) & =\mathcal{S}\left(\frac{1}{2} \exp ^{\diamond}\left(-\frac{1}{2} W_{\phi}(x)\right)\right)(\xi) \cdot \mathcal{S}\left(\hat{\mathrm{E}}^{x}\left[\int_{0}^{\tau_{\mathcal{D}}} \cdots d t\right]\right)(\xi) \\
& =\frac{1}{2} \exp \left(-\frac{1}{2}\left(\phi_{x}, \xi\right)\right) \cdot \hat{\mathrm{E}}^{x}\left[\mathcal{S}\left(\int_{0}^{\tau_{\mathcal{D}}} \cdots d t\right)(\xi)\right] .
\end{aligned}
$$

using the integrability of the integrand, the result about Bochner integrability from Theorem 4.1, and (2.13). Now, recall that the exponent in the last integral can be written as in (4.8). This gives

$$
\mathcal{S}\left(\int_{0}^{\tau_{\mathcal{D}}} f\left(b_{t}\right) \diamond \exp ^{\circ}\left[-I_{1}\left(\phi_{t}^{(1)}\right)-I_{2}\left(\phi_{t}^{(2)}\right)\right] d t\right)(\xi)=\int_{0}^{\tau_{\mathcal{D}}} \mathcal{S} f\left(b_{t}\right)(\xi) \cdot \exp \left[\left(\eta_{1}, \xi\right)+\left(\eta_{2}, \xi^{\widehat{\otimes}^{2}}\right)\right] d t
$$

using Lemma 4.2 , and setting $\eta_{1}=-\phi_{t}^{(1)}, \eta_{2}=-\phi_{t}^{(2)}$. By expanding the exponential we get

$$
\begin{aligned}
\exp \left[\left(\eta_{1}, \xi\right)+\left(\eta_{2}, \xi^{\widehat{\otimes} 2}\right)\right] & =\sum_{n, m=0}^{\infty} \frac{1}{n ! m !}\left(\eta_{1}, \xi\right)^{n}\left(\eta_{2}, \xi^{\widehat{\otimes} 2}\right)^{m} \\
& =\sum_{k=0}^{\infty} \sum_{n+2 m=k} \frac{1}{n ! m !}\left(\eta_{1}, \xi\right)^{n}\left(\eta_{2}, \xi^{\widehat{\otimes} 2}\right)^{m} \\
& =\sum_{k=0}^{\infty} \sum_{m=0}^{\lfloor k / 2\rfloor} \frac{1}{(k-2 m) ! m !}\left(\eta_{1}, \xi\right)^{k-2 m}\left(\eta_{2}, \xi^{\widehat{\otimes} 2}\right)^{m}
\end{aligned}
$$

By using the definition of the symmetric product, it is not hard to see that each of the terms in the last sum can be written as

$$
\frac{1}{(k-2 m) ! m !}\left(\eta_{1}^{\widehat{\otimes} k-2 m} \widehat{\otimes} \eta_{2}^{\widehat{\otimes} m}, \xi^{\hat{\otimes} n}\right)
$$

thus

$$
\exp \left[\left(\eta_{1}, \xi\right)+\left(\eta_{2}, \xi^{\bar{\otimes} 2}\right)\right]=\sum_{k=0}^{\infty}\left(\theta^{(k)}, \xi^{\bar{\otimes} k}\right)
$$

where

$$
\theta^{(k)}=\sum_{m=0}^{\lfloor k / 2\rfloor} \frac{1}{(k-2 m) ! m !} \eta_{1}^{\widehat{\otimes} k-2 m} \widehat{\otimes} \eta_{2}^{\widehat{\otimes} m} .
$$

Next, we multiply with $\mathcal{S} f\left(b_{t}\right)(\xi)=\sum_{l=0}^{\infty}\left(f_{b_{t}}^{(l)}, \xi^{\widehat{\otimes} l}\right)$ and again by using the definition of symmetric product we get

$$
\begin{aligned}
\mathcal{S} f\left(b_{t}\right)(\xi) \cdot \exp \left[\left(\eta_{1}, \xi\right)+\left(\eta_{2}, \xi^{\widehat{\otimes} 2}\right)\right] & =\sum_{k, l=0}^{\infty}\left(f_{b_{t}}^{(l)}, \xi^{\widehat{\otimes} l}\right)\left(\theta^{(k)}, \xi^{\widehat{\otimes} k}\right) \\
& =\sum_{n=0}^{\infty} \sum_{k=0}^{n}\left(f_{b_{t}}^{(n-k)}, \xi^{\widehat{\otimes} n-k}\right)\left(\theta^{(k)}, \xi^{\widehat{\otimes} k}\right) \\
& =\sum_{n=0}^{\infty}\left(\psi^{(n)}, \xi^{\widehat{\otimes} n}\right)
\end{aligned}
$$

where

$$
\psi^{(n)}=\sum_{k=0}^{n} \sum_{m=0}^{\lfloor k / 2\rfloor} \frac{1}{(k-2 m) ! m !} f_{b_{t}}^{(n-k)} \widehat{\otimes} \eta_{1}^{\widehat{\otimes} k-2 m} \widehat{\otimes} \eta_{2}^{\widehat{\otimes} m}
$$


and $f_{b_{t}}^{(l)}$ denotes the $l$ th chaos of $f\left(b_{t}\right)$. Integrating over $\left[0, \tau_{\mathcal{D}}\right]$ and taking the expectation with respect to $\hat{P}_{x}$ gives

$$
\hat{\mathrm{E}}^{x}\left[\mathcal{S}\left(\int_{0}^{\tau_{\mathcal{D}}} \cdots d t\right)(\xi)\right]=\sum_{n=0}^{\infty}\left(\hat{\mathrm{E}}^{x}\left[\int_{0}^{\tau_{\mathcal{D}}} \psi^{(n)} d t\right], \xi^{\widehat{\otimes} n}\right)
$$

using the absolute convergence of the $\mathcal{S}$-transform and Fubinis theorem twice. Finally, multiplying with $\frac{1}{2} \exp \left(-\frac{1}{2}\left(\phi_{x}, \xi\right)\right)$ gives

$$
\begin{aligned}
S p_{\phi}(x)(\xi)= & \frac{1}{2} \sum_{k, l=0}^{\infty} \frac{(-1)^{k}}{k ! 2^{k}}\left(\phi_{x}^{\widehat{\otimes} k}, \xi^{\widehat{\otimes} k}\right)\left(\hat{\mathrm{E}}^{x}\left[\int_{0}^{\tau_{\mathcal{D}}} \psi^{(l)} d t\right], \xi^{\widehat{\otimes} l}\right) \\
& =\sum_{n=0}^{\infty}\left(\sum_{l=0}^{n} \frac{(-1)^{n-l}}{(n-l) ! 2^{n-l}} \phi_{x}^{\widehat{\otimes} n-l} \widehat{\otimes}^{x}\left[\int_{0}^{\tau_{\mathcal{D}}} \psi^{(l)} d t\right], \xi^{\widehat{\otimes} n}\right) .
\end{aligned}
$$

And the result follows by uniqueness of the $\mathcal{S}$-transform.

We see from Th. 5.2 that the chaos kernels $p_{\phi}^{(n)}(x ; \cdot)$ are regular functions. In fact, from the previous section we know that $p_{\phi}^{(n)}(x ; \cdot) \in \hat{L_{n}^{2}}$.

\section{STABILITY WITH RESPECT TO DATA}

In this section we show that $p_{\phi}(x)$ is continuous with respect to both $f$ and $\phi$ when chosing appropriate norms. Such stability results are of importance in real applications.

We have the following result following directly from the proof of Theorem 4.1:

Proposition 6.1. Denote by $p_{\phi}(f ; x)$ the solution of the pressure equation (3.1) with source $f$. Suppose $f, g \in \mathcal{G}_{-q_{s}}^{-1}$ with norms uniformly bounded in $x \in \mathcal{D}$. Then

$$
\left\|p_{\phi}(f ; \cdot)-p_{\phi}(g ; \cdot)\right\|_{-1,-q, \infty} \leq K\|f-g\|_{-1,-q_{*}, \infty}
$$

for $q>\max \left(q_{s}, q_{\rho}\right)$ with $q_{\rho}$ defined as in Theorem 4.1, and where $K$ is a positive constant independent of $f$ and $g$.

Proof. The proposition follows by the same arguments as in the proof of Theorem 4.1 using the linearity of the solution.

Consider now the mapping $\phi \rightarrow p_{\phi}(x)$ :

Proposition 6.2. Let $\phi, \psi \in \mathcal{S}\left(\mathbb{R}^{d}\right), q_{0}=\max \left(q_{f}, q_{\rho}+\ln 2\right)$, and suppose the assumptions of Theorem 4.1 holds. Then for $q>q_{0}$

$$
\left\|p_{\phi}-p_{\psi}\right\|_{-1,-q, \infty} \leq K\left\{|\phi-\psi|_{2}+|\nabla \phi-\nabla \psi|_{2}^{2}+|\Delta \phi-\Delta \psi|_{2}\right\}
$$

for some positive constant $K$ dependent on $\phi$ and $\psi$ (but not on the difference).

Before we proceed to give the proof, we need the following intermediate result:

Lemma 6.3. For any $q, r \in \mathbb{N}_{0}$ let $f, g \in \widehat{L_{q}^{2}}$ and $h \in \widehat{L_{r}^{2}}$. Then

$$
|f \widehat{\otimes} g|_{\widehat{L_{q+r}^{2}}} \leq|f|_{\widehat{L_{q}^{2}}}|g|_{\widehat{L_{r}^{2}}}
$$

and for any $m \in \mathbb{N}$ we have

$$
\left|f^{\widehat{\otimes} m}-g^{\widehat{\otimes} m}\right|_{\widehat{L_{q m}^{2}}} \leq m K^{m-1}|f-g|_{\widehat{L_{q}^{2}}}
$$

where $K=\max \left(|f|_{\widehat{L_{q}^{2}}},|g|_{\overparen{L_{q}^{2}}}\right)$. 
Proof. The proof of (6.3) follows by direct calculation. The key point is to use Cauchy-Schwarz' inequality for each term in the norm expression. The estimate in (6.4) follows by (6.3) and induction on $m$.

Proof of Proposition 6.2. The proof goes by a direct estimation of the norms. First note that we can write

$$
\begin{aligned}
p_{\phi}(x)-p_{\psi}(x)= & \frac{1}{2}\left(\exp ^{\circ}\left(-\frac{1}{2} W_{\phi}(x)\right)-\exp ^{\circ}\left(-\frac{1}{2} W_{\psi}(x)\right)\right) \diamond \hat{\mathrm{E}}^{x}\left[\int_{0}^{\tau_{\mathcal{D}}} f\left(b_{t}\right) \diamond X_{\phi}(t) d t\right] \\
& +\frac{1}{2} \exp ^{\circ}\left(-\frac{1}{2} W_{\psi}(x)\right) \diamond \hat{\mathrm{E}}^{x}\left[\int_{0}^{\tau_{\mathcal{D}}} f\left(b_{t}\right) \diamond\left(X_{\phi}(t)-X_{\psi}(t)\right) d t\right]
\end{aligned}
$$

where

$$
X_{\phi}(t)=\exp ^{\circ}\left(-I_{1}\left(\phi_{t}^{(1)}\right)-I_{2}\left(\phi_{t}^{(2)}\right)\right),
$$

and with the obvious definition for $X_{\psi}$. By the triangle inequality and the norm estimate in Proposition 2.3 we get (for $q>q_{1}>q_{0}$ )

$$
\begin{aligned}
\left\|p_{\phi}(x)-p_{\psi}(x)\right\|_{-1,-q} \leq & C_{1}\left\|\exp ^{\diamond}\left(-\frac{1}{2} W_{\phi}(x)\right)-\exp ^{\circ}\left(-\frac{1}{2} W_{\psi}(x)\right)\right\|_{-1,0} \cdot \\
& \left\|\hat{\mathrm{E}}^{x}\left[\int_{0}^{\tau_{\mathcal{D}}} f\left(b_{t}\right) \diamond X_{\phi}(t) d t\right]\right\|_{-1,-q_{1}} \\
+ & C_{1}\left\|\exp ^{\diamond}\left(-\frac{1}{2} W_{\psi}(x)\right)\right\|_{-1,0} \cdot\left\|\hat{\mathrm{E}}^{x}\left[\int_{0}^{\tau_{\mathcal{D}}} f\left(b_{t}\right) \diamond\left(X_{\phi}(t)-X_{\psi}(t)\right) d t\right]\right\|_{-1,-q_{1}}
\end{aligned}
$$

where $C_{1}=\left(1-e^{-\left(q-q_{1}\right)}\right)^{-1}$. There are four norms that we need to estimate. We start with the left-most norm. Using the definition of the Wick exponential and Lemma 6.3 we get

$$
\begin{aligned}
\| \exp ^{\circ}\left(-\frac{1}{2} W_{\phi}(x)\right) & -\exp ^{\circ}\left(-\frac{1}{2} W_{\psi}(x)\right)\left\|_{-1,0}^{2}=\right\| \sum_{n=1}^{\infty} \frac{1}{n !} I_{n}\left(\left(-\frac{1}{2} \phi_{x}\right)^{\widehat{\otimes} n}-\left(-\frac{1}{2} \psi_{x}\right)^{\widehat{\otimes} n}\right) \|_{-1,0}^{2} \\
& =\sum_{n=1}^{\infty} \frac{1}{(n !)^{2}} 4^{-n}\left|\phi_{x}^{\widehat{\otimes} n}-\psi_{x}^{\widehat{\otimes} n}\right|_{2}^{2} \leq \sum_{n=1}^{\infty} \frac{1}{(n !)^{2}} 4^{-n} n^{2} K_{1}^{2(n-1)}|\phi-\psi|_{2}^{2} \\
& =\frac{1}{4}|\phi-\psi|_{2}^{2} \sum_{n=0}^{\infty} \frac{\left(K_{1}^{2} / 4\right)^{n}}{(n !)^{2}}=\frac{1}{4} B_{0}\left(K_{1} / \sqrt{2}\right)|\phi-\psi|_{2}^{2}
\end{aligned}
$$

where $K_{1}:=\max (|\phi|,|\psi|)$ and $B_{0}$ is the modified Bessel function. From the proof of Theorem 4.1 we have

$$
\left\|\hat{\mathrm{E}}^{x}\left[\int_{0}^{\tau_{\mathcal{D}}} f\left(b_{t}\right) \diamond X_{\phi}(t) d t\right]\right\|_{-1,-q_{1}} \leq K_{2}
$$

for a positive constant $K_{2}$. For the third norm note that by Example 2.1 we have

$$
\left\|\exp ^{\diamond}\left(W_{\psi}\right)\right\|_{-1,0}=\exp \left(|\psi|_{2} / 2\right) .
$$

Finally we estimate the forth norm. Bochner integrability yields,

$$
\begin{aligned}
\| \hat{\mathrm{E}}^{x}\left[\int_{0}^{\tau_{\mathcal{D}}} f\left(b_{t}\right)\right. & \left.\propto\left\{X_{\phi}(t)-X_{\psi}(t)\right\} d t\right] \|_{-1,-q_{1}} \\
& \leq C_{2}\|f\|_{-1,-q_{f}, \infty} \hat{\mathrm{E}}^{x}\left[\int_{0}^{\tau_{\mathcal{D}}}\left\|X_{\phi}(t)-X_{\psi}(t)\right\|_{-1,-q_{2}} d t\right]
\end{aligned}
$$

where $q_{2}$ is a constant such that $q_{1}>q_{2}>q_{0}$ and $C_{2}=\left(1-e^{-\left(q_{1}-q_{2}\right)}\right)^{-1}$. Consider the norm inside the expectation. For any $p, r \in\left(q_{0}, q_{2}\right)$ we get

$$
\begin{aligned}
\left\|X_{\phi}(t)-X_{\psi}(t)\right\|_{-1,-q_{2}} \leq & C_{3}\left\|\exp ^{\diamond}\left(-I_{1}\left(\phi_{t}^{(1)}\right)\right)\right\|_{-1,-p}\left\|\exp ^{\diamond}\left(-I_{2}\left(\phi_{t}^{(2)}\right)\right)-\exp ^{\diamond}\left(-I_{2}\left(\psi_{t}^{(2)}\right)\right)\right\|_{-1,-r} \\
& +C_{3}\left\|\exp ^{\diamond}\left(-I_{2}\left(\psi_{t}^{(2)}\right)\right)\right\|_{-1,-r}\left\|\exp ^{\diamond}\left(-I_{1}\left(\phi_{t}^{(1)}\right)\right)-\exp ^{\diamond}\left(-I_{1}\left(\psi_{t}^{(1)}\right)\right)\right\|_{-1,-p}
\end{aligned}
$$


where $C_{3}=\left(1-e^{-\left(q_{2}-\max (p, r)\right)}\right)^{-1}$. But,

$$
\begin{aligned}
\left\|\exp ^{\diamond}\left(-I_{1}\left(\phi_{t}^{(1)}\right)\right)-\exp ^{\diamond}\left(-I_{1}\left(\psi_{t}^{(1)}\right)\right)\right\|_{-1,-p} & \leq\left\|\sum_{n=1}^{\infty} I_{n}\left(\frac{1}{n !}\left(\left(-\phi_{t}^{(1)}\right)^{\hat{\otimes} n}-\left(-\psi_{t}^{(1)}\right)^{\widehat{\otimes} n}\right)\right)\right\|_{-1,-p} \\
& \leq \sum_{n=1}^{\infty} \frac{e^{-n p}}{n !}\left|\phi_{t}^{(1) \hat{\otimes} n}-\psi_{t}^{(1) \hat{\otimes} n}\right|_{2} .
\end{aligned}
$$

From Lemma 6.3 we have

$$
\left|\phi_{t}^{(1) \otimes} n-\psi_{t}^{(1) \otimes \bar{\otimes} n}\right|_{2} \leq n\left(\max \left(\left|\phi_{t}^{(1)}\right|_{2},\left|\psi_{t}^{(1)}\right|_{2}\right)\right)^{n-1}\left|\phi_{t}^{(1)}-\psi_{t}^{(1)}\right|_{2} .
$$

Using the norm estimate (4.12) we get

$$
\left\|\exp ^{\diamond}\left(-I_{1}\left(\phi_{t}^{(1)}\right)\right)-\exp ^{\diamond}\left(-I_{1}\left(\psi_{t}^{(1)}\right)\right)\right\|_{-1,-p} \leq e^{-p} \exp \left(e^{-p} K_{4 a}(t)\right)\left(\frac{1}{2}|\phi-\psi|_{2}+\frac{t}{4}|\Delta \phi-\Delta \psi|_{2}\right)
$$

for

$$
K_{4 a}(t)=\frac{1}{2} \max \left(|\phi|_{2},|\psi|_{2}\right)+\frac{t}{4} \max \left(|\Delta \phi|_{2},|\Delta \psi|_{2}\right) .
$$

Equivalently, using the norm estimate (4.9) we get

$$
\left\|\exp ^{\circ}\left(-I_{2}\left(\phi_{t}^{(2)}\right)\right)-\exp ^{\circ}\left(-I_{2}\left(\psi_{t}^{(2)}\right)\right)\right\|_{-1,-r} \leq e^{-2 r} \exp \left(e^{-2 r} K_{4 b}(t)\right) \frac{t}{8}|\nabla \phi-\nabla \psi|_{2}^{2}
$$

where

$$
K_{4 b}(t)=\frac{t}{8} \max \left(|\nabla \phi|_{2}^{2},|\nabla \psi|_{2}^{2}\right) .
$$

Collecting these two estimates and using (4.10) and (4.11) give the bound

$$
\left\|X_{\phi}(t)-X_{\psi}(t)\right\|_{-1,-q_{2}} \leq a e^{\hat{\rho} t}+b t e^{\hat{\rho} t}
$$

where $C_{4}=C_{3} \exp \left(\max \left(|\phi|_{2},|\psi|_{2}\right)\right), a=C_{4}|\phi-\psi|_{2}, b=C_{4}\left(|\Delta \phi-\Delta \psi|_{2}+|\nabla \phi-\nabla \psi|_{2}^{2}\right)$ and

$$
\hat{\rho}=e^{-p} \frac{1}{4} \max \left(|\Delta \phi|_{2},|\Delta \psi|_{2}\right)+e^{-2 r} \frac{1}{8} \max \left(|\nabla \phi|_{2}^{2},|\nabla \psi|_{2}^{2}\right) .
$$

If $\hat{\rho}=0$ we are finished. Assume $\hat{\rho}>0$ and integrate to get

$$
\hat{\mathrm{E}}^{x}\left[\int_{0}^{\tau_{\mathcal{D}}}\left\|X_{\phi}(t)-X_{\psi}(t)\right\|_{-1,-q_{2}} d t\right] \leq \frac{1}{\hat{\rho}^{2}}\left(b-\hat{\rho} a+(\hat{\rho} a-b) \hat{\mathrm{E}}^{x}\left[e^{\hat{\rho} \tau_{\mathcal{D}}}\right]+\hat{\rho} b \hat{\mathrm{E}}^{x}\left[\tau_{\mathcal{D}} e^{\hat{\rho} \tau_{\mathcal{D}}}\right]\right) .
$$

Note that by asumption on $p$ and $r$ we have $\hat{\rho}<\rho$, where $\rho$ is the constant from the estimate in (4.1). This explaines the added $\ln 2$ in the assumption on $q_{0}$. Using e.g. Hölders inequality it is easy to show that uniform boundedness of $\hat{\mathrm{E}}^{x}\left[e^{\rho \tau_{\mathcal{D}}}\right]$ implies uniform boudedness of $\hat{\mathrm{E}}^{x}\left[\tau_{\mathcal{D}} e^{\hat{\rho} \tau_{\mathcal{D}}}\right]$. The proposition is proved.

\section{THE WICK PRODUCT AND TRANSLATION}

In this last section we shall prove an explicit connection between a stochastic pressure equation with ordinary product and a Wick-type equation when $d=1$. The boundary values of the problem will be slightly changed, to have a more transparent and easy derivation of the connection. Instead of using Dirichlet conditions, we use combined Dirichlet and Neumann conditions. It is shown that the solutions may be written as stochastic translations of each other, where the translation is taken in the direction of the smearing function of $\phi$. We believe that such a connection may be of some use in evaluating which model, Wick or ordinary product, to use in real applications. In deriving this connection, we will assume existence of sufficiently regular solutions of the two problems. A similar connection may be shown for (3.1) with Dirichlet conditions when $d=1$. All these questions will be considered at a more rigourous and complete level in a future paper.

The derivation is based on some interesting relations between translation and the Wick product first presented in Benth and Gjessing [6] and Benth [2] for the case $b=0$. In this section we will generalize these results to the spaces $\mathcal{G}^{-b}$. We want to emphasis that such results have a wider range of applications than presented in this paper. For example, in connection with quasi-linear 
ordinary stochastic differential equations Gjessing [10] proved interesting formulas for the solution using Wick product and translation.

We start by extending some results on translation from Potthoff and Timpel [18]: The following lemma is a direct generalization of the corresponding result for the space $\mathcal{G}$ given in Potthoff and. Timpel [18].

Lemma 7.1. Let $b \in[0,1], h \in L^{2}\left(\mathbb{R}^{d}\right)$ and for $\phi \in \mathcal{G}^{b}$ define the translation map $\tau_{h}$ by $\phi(\omega) \mapsto$ $\phi(\omega+h)$. Then $\tau_{h}$ is a continuous linear map from $\mathcal{G}^{b}$ to $\mathcal{G}^{b}$.

Proof. The proof follows the lines of [18]. Linearity is obvious. We need to establish the continuity. Let $\phi=\sum_{n=0}^{N} I_{n}\left(\phi^{(n)}\right)$ be in $\mathcal{P}$ and recall from Hida et al. [12] the formula

$$
\phi(\omega+h)=\sum_{n=0}^{N} \sum_{k=0}^{n}\left(\begin{array}{l}
n \\
k
\end{array}\right) I_{k}\left(\phi^{(n, n-k)}\right)
$$

where

$$
\phi^{(n, n-k)}(u):=\int_{\mathbb{R}^{d(n-k)}} \phi^{(n)}(u, v) h^{\hat{\otimes} n-k}(v) d v .
$$

Note that in Hida et al. [12] this formula is given for $d=1$ but the extension to $d \in \mathbb{N}$ is straigtforward. Then using the triangle inequality, the definition of the norm, Cauchy-Schwarz' inequality and the Binomial formula we get

$$
\begin{aligned}
\|\phi(\cdot+h)\|_{b, q} & \leq \sum_{n=0}^{N} \sum_{k=0}^{n}\left\|\left(\begin{array}{l}
n \\
k
\end{array}\right) I_{k}\left(\phi^{(n, n-k)}\right)\right\|_{b, q} \\
& \leq \sum_{n=0}^{N} \sum_{k=0}^{n}\left(\begin{array}{l}
n \\
k
\end{array}\right)(k !)^{(b+1) / 2} e^{q k}|h|_{2}^{n-k}\left|\phi^{(n)}\right|_{2} \\
& \leq \sum_{n=0}^{N}\left|\phi^{(n)}\right|_{2}(n !)^{(b+1) / 2} e^{q n} \sum_{k=0}^{n}\left(\begin{array}{l}
n \\
k
\end{array}\right) e^{-q(n-k)}|h|_{2}^{n-k} \\
& =\sum_{n=0}^{N}\left|\phi^{(n)}\right|_{2}(n !)^{(b+1) / 2} e^{q_{0} n}
\end{aligned}
$$

where $q_{0}=q+\ln \left(1+e^{-q}|h|_{2}\right)$. Thus for any $q^{\prime}>q_{0}$ we have by Cauchy-Schwarz that

$$
\begin{aligned}
\|\phi(\cdot+h)\|_{b, q} & \leq\left(\sum_{n=0}^{N} e^{2\left(q_{0}-q^{\prime}\right)}\right)^{1 / 2}\left(\sum_{n=0}^{N}(n !)^{(b+1)} e^{2 q n}\left|\phi^{(n)}\right|_{2}^{2}\right)^{1 / 2} \\
& \leq\left(1-e^{2\left(q_{0}-q^{\prime}\right)}\right)^{-1 / 2}\|\phi\|_{b, q^{\prime}} .
\end{aligned}
$$

The lemma follows by extending $\tau_{h}$ continuously to $\mathcal{G}^{b}$.

Definition 7.1. Let $h \in L^{2}\left(\mathbb{R}^{d}\right)$ and $b \in[0,1]$. Then the adjoint operator $\tau_{h}^{*}: \mathcal{G}^{-b} \mapsto \mathcal{G}^{-b}$ is defined as

$$
\left\langle\left\langle\tau_{h}^{*} \Phi, \phi\right\rangle\right\rangle=\left\langle\left\langle\Phi, \tau_{h} \phi\right\rangle\right\rangle
$$

for all $\phi \in \mathcal{G}^{b}$.

Remark. It follows from Lemma 7.1 that $\tau_{h}^{*}$ is a continuous linear map from $\mathcal{G}^{-b}$ to $\mathcal{G}^{-b}$.

As for $b=0$ in Benth and Gjessing [6] and Benth [2] we have

Lemma 7.2. Let $h \in L^{2}\left(\mathbb{R}^{d}\right), b \in[0,1]$ and $\Phi \in \mathcal{G}^{-b}$. Then

$$
\tau_{h}^{*} \Phi=\exp ^{\diamond}\left(W_{h}\right) \diamond \Phi .
$$


Proof. Take the $\mathcal{S}$-transform. For any $\eta \in \mathcal{S}\left(\mathbb{R}^{d}\right)$ (if $b=1$ we restrict to those $\eta$ with $e^{q}|\eta|_{2}<1$ for sufficiently big $q>0$ ) we have

$$
\mathcal{S}\left(\tau_{h}^{*} \Phi\right)(\eta)=\left\langle\left\langle\tau_{h}^{*} \Phi, \exp ^{\diamond}\left(W_{\eta}\right)\right\rangle\right\rangle=\left\langle\left\langle\Phi, \tau_{h} \exp ^{\diamond}\left(W_{\eta}\right)\right\rangle\right\rangle=\mathcal{S}\left(\exp \left(W_{h}\right)\right)(\eta) \cdot \mathcal{S} \Phi(\eta)
$$

since $\tau_{h} \exp ^{\diamond}\left(W_{\eta}\right)=\exp (h, \eta) \exp ^{\diamond}\left(W_{\eta}\right)$. Uniqueness of the $\mathcal{S}$-transform completes the proof.

Inspired by Benth and Gjessing [6] and Benth [2] we introduce a generalization of the translation operator. The only essential difference from what was done in Benth and Gjessing [6] and Benth [2] is for the case $b=1$. Extra care must be taken in this case because of the smoothness properties of the Wick exponentials.

Definition 7.2. Let $h \in L^{2}\left(\mathbb{R}^{d}\right), b \in[0,1)$. For any $\Phi \in \mathcal{G}^{-b}$ define the generalized translation $T_{h} \Phi$ as

$$
\left\langle\left\langle T_{h} \Phi, \phi\right\rangle\right\rangle=\left\langle\left\langle\Phi, \tau_{h}^{*} \phi\right\rangle\right\rangle
$$

for all $\phi \in \mathcal{G}^{b}$.

Note the relation (7.1) and that $\exp ^{\circ} W_{h} \in \mathcal{G}^{b}$ for all $b \in[0,1)$ and $h \in L^{2}\left(\mathbb{R}^{d}\right)$. Thus, the above generalized translation is a well-defined, continouous and linear map from $\mathcal{G}^{-b}$ into $\mathcal{G}^{-b}$ by Theorem 2.5. Now, consider the slightly more difficult case $b=1$ :

Lemma 7.3. Assume $\Phi \in \mathcal{G}_{-q}^{-1}$ for some $q \geq 0$. Let $p>q+\ln 2$ and suppose $h \in L^{2}\left(\mathbb{R}^{d}\right)$ with $e^{\mathcal{P}}|h|_{2}<1$. Then $T_{h} \Phi \in \mathcal{G}_{-p}^{-1}$ and

$$
\left\|T_{h} \Phi\right\|_{-1,-p} \leq 2\left\|\exp ^{\curvearrowright}\left(W_{h}\right)\right\|_{1, p}\|\Phi\|_{-1,-q} .
$$

Proof. Given $\phi \in \mathcal{G}_{p}^{1}$ and $\Phi \in \mathcal{G}_{-q}^{-1}$, then using Lemma 7.2, Cauchy-Schwarz' inequality and Corollary 2.4 we get

$$
\begin{aligned}
\left|\left\langle\left\langle T_{h} \Phi, \phi\right\rangle\right\rangle\right| & =\left|\left\langle\left\langle\Phi, \tau_{h}^{*} \phi\right\rangle\right\rangle\right| \\
& =\left|\left\langle\left\langle\Phi, \exp ^{\diamond}\left(W_{h}\right) \diamond \phi\right\rangle\right\rangle\right| \\
& \leq\|\Phi\|_{-1,-q}\left\|\exp ^{\circ}\left(W_{h}\right) \diamond \phi\right\|_{1, q} \\
& \leq\|\Phi\|_{-1,-q}\left(1-e^{q-p}\right)^{-1}\left\|\exp ^{\circ}\left(W_{h}\right)\right\|_{1, p}\|\phi\|_{1, p}
\end{aligned}
$$

and the lemma follows.

We see that when $b=1, T_{h}$ does not define a continuous and linear map from $\mathcal{G}^{-1}$ into $\mathcal{G}^{-1}$ since we cannot find a function $h$ (except for the trivial $h(x)=0$ a.e. $x$ ) such that $e^{\mathcal{P}}|h|_{2}<1$ for general $p$. But we can define $T_{h}$ as a continuous and linear map from $\mathcal{G}_{-q}^{-1}$ into $\mathcal{G}_{-p}^{-1}$ for a class of functions $h$, where $p>q+\ln 2$.

We are now ready to generalize a relation first given in Gjessing [10] in a Wiener space setting and later generalized for $b=0$ in the white noise case in Benth and Gjessing [6] and Benth [2].

Lemma 7.4. Let $h \in L^{2}\left(\mathbb{R}^{d}\right), b \in[0,1)$ and $\Phi \in \mathcal{G}^{-b}$. Then

$$
\exp ^{\diamond}\left(W_{h}\right) \diamond \Phi=\exp ^{\diamond}\left(W_{h}\right) \cdot T_{-h} \Phi .
$$

If $b=1,(7.3)$ holds for all $h \in L^{2}\left(\mathbb{R}^{d}\right)$ such that $e^{p}|h|_{2}<1$, where $p>q+\ln 2$ and $\Phi \in \mathcal{G}_{-q}^{-1}$.

Proof. The proof for $b \in[0,1)$ follows the ideas in Benth and Gjessing [6] and Benth [2]. First notice that from Lemma 7.2 we have

$$
\left\langle\left\langle\Phi,-\tau_{h} \phi\right\rangle\right\rangle=\left\langle\left\langle\exp ^{\diamond}\left(W_{h}\right) \diamond \Phi, \phi\right\rangle\right\rangle . \quad\left(\phi \in \mathcal{G}^{b}\right)
$$

Setting $\Phi=1$ gives a Cameron-Martin-Girsanov formula

$$
\int \phi(\omega+h) d \mu(\omega)=\int \exp ^{\curvearrowright}\left(E_{h}\right)(\omega) \phi(\omega) d \mu(\omega)
$$


For $\Phi \in \mathcal{G}^{b}$ we get

$$
\begin{aligned}
\int\left(\exp ^{\circ}\left(W_{h}\right) \diamond \Phi\right)(\omega) \phi(\omega) d \mu(\omega) & =\int \Phi(\omega) \phi(\omega+h) d \mu(\omega) \\
& =\int \Phi(\omega-h+h) \phi(\omega+h) d \mu(\omega) \\
& =\int \exp ^{\diamond}\left(W_{h}\right)(\omega) \Phi(\omega-h) \phi(\omega) d \mu(\omega)
\end{aligned}
$$

Generalizing to $\Phi \in \mathcal{G}^{-b}$. gives

$$
\left\langle\left\langle\exp ^{\diamond}\left(W_{h}\right) \diamond \Phi, \phi\right\rangle\right\rangle=\left\langle\left\langle T_{-h} \Phi \cdot \exp ^{\diamond}\left(W_{h}\right), \phi\right\rangle\right\rangle
$$

for all $\phi \in \mathcal{G}^{b}$. The right hand side is understood in the usual sence

$$
\left\langle\left\langle T_{-h} \Phi \cdot \exp ^{\circ}(\langle\cdot, h\rangle), \phi\right\rangle\right\rangle=\left\langle\left\langle T_{-h} \Phi, \exp ^{\circ}(\langle\cdot, h\rangle) \cdot \phi\right\rangle\right\rangle
$$

and is well-defined as a consequence of Theorem 2.7. Thus the first part of the lemma is established. We continue the proof for $b=1$. Note that Lemma 7.2 holds also for $b=1$. If we assume $\Phi \in \mathcal{G}_{-q}^{-1}$ and $|h|_{2} e^{p}<1$ for a $p>q+\ln 2$, then we can perform the same calculations as for the $b<1$ case. Thus it is clear that (7.4) holds for any $\phi \in \mathcal{G}_{p}^{1}$ with $p>q+\ln 2$.

We will in the rest of the section consider the pressure equation in dimension one, that is $d=1$. As mentioned above, we shall treat slightly different initial conditions than in (3.1) which makes the calculations much simpler. Let $\mathcal{D}=[a, b]$ for $a>b$. Let $p(x)$ denote the solution of the pressure equation of Wick-type (the solution of $(3.1)$ ), but now with boundary condition $p(a)=0$ and $p^{\prime}(a)=k$ where $k$ is a deterministic constant, and let $q(x)$ be the solution when we model with ordinary product instead, i.e.;

$$
\begin{gathered}
-\frac{d}{d x}\left(\exp ^{\diamond} W_{\phi}(x) \cdot q^{\prime}(x)\right)=f \text { on }(a, b), \\
q(a)=0, q^{\prime}(a)=k .
\end{gathered}
$$

Using Lemma 7.2 we can show that any Wick solution $p(x)$ can be written in terms of the product solution $q(x)$, and vice-versa:

Theorem 7.5. Let $\phi \in \mathcal{S}(\mathbb{R})$. Assume that $p(x) \in \mathcal{G}^{-1}$ is differentiable in $x, p^{\prime}(x) \in \mathcal{G}_{-q}^{-1}$ (with norm uniformly bounded in $x$ ) and $T_{-\phi_{y}} p^{\prime}(y)$ is Bochner integrable on $[a, b]$ for $\phi$ such that $e^{p}|\phi|_{2}<1$ and $p>q+\ln 2$. Then

$$
q(x)=\int_{a}^{x} T_{-\phi_{y}} p^{\prime}(y) d y .
$$

On the other hand, assume that $q(x) \in \mathcal{G}^{-1}$ is differentiable in $x, q^{\prime}(x) \in \mathcal{G}_{-q}^{-1}$ (uniformly in $x$ ) and $T_{\phi_{y}} q^{\prime}(y)$ is Bochner integrable on $[a, b]$ for $\phi$ such that $e^{p}|\phi|_{2}<1$ and $p>q+\ln 2$. Then

$$
p(x)=\int_{a}^{x} T_{\phi_{y}} q^{\prime}(y) d y .
$$

Proof. We start by showing that the boundary values for the two problems are satisfied: Obviously, $q(a)=p(a)=0$. Using the definition of $q(x)$, we have $q^{\prime}(a)=T_{-\phi_{a}} p^{\prime}(a)=T_{-\phi_{1}} k=k$. Similarly we get $p^{\prime}(a)=T_{\phi_{a}} q^{\prime}(a)=T_{\phi_{a}} k=k$ when $p(x)$ is defined through the solution of the pressure equation with ordinary product.

In order to show the first part of the Theorem we use the generalized formula (7.3) and reformulate the Wick equation to a product equation:

$$
\begin{aligned}
\frac{d}{d x}\left(\exp ^{\diamond} \widehat{\left.W_{\phi_{x}} \diamond p^{\prime}(x)\right)}\right. & =\frac{d}{d x}\left(\exp ^{\diamond} W_{\phi_{x}} \cdot T_{-\phi_{x}} p^{\prime}(x)\right) \\
& =\frac{d}{d x}\left(\exp ^{\diamond} W_{\phi_{x}} \cdot \frac{d}{d x} \int_{a}^{x} T_{-\phi_{y}} p^{\prime}(y) d y\right)
\end{aligned}
$$


where the last equality follows from the Fundamental Theorem of Calculus.

This same calculation can be reversed to show the other way of the connection.

We would like to remark that it is possible to calculate an explicit solution $p(x)$ having the desired regularity properties using Wick calculus (see Kondratiev, Leukert and Streit [14] and Holden et al. [13]). In fact, Holden et al. [13] calculate an explicit solution when the Neumann condition has a special stochastic form and the source $f$ is zero: Introduce the boundary conditions $p(a)=1, \exp ^{\diamond}\left(W_{h}\right) \diamond p^{\prime}(a)=k$ and $q(a)=1, \exp ^{\diamond}\left(W_{h}\right) q^{\prime}(a)=k$ for the two models. Then it follows by a simple calculation using generalized translation on their explicit solution that $p(x)=q(x) \cdot \exp \left(|h|^{2}\right)$, which coincides with the formula (3.5.11) in Holden et al. [13]. Note that we have used a general source $f$ possibly being a random field in Theorem 7.5.

\section{REFERENCES}

[1] F.E. Benth. Integrals in the Hida distribution space. Stochastic Analysis and Related Topics; Stochastic Monographs Vol. 8. T. Lindstrøm, B. Øksendal og A. S. Ustunel (editors). Gordon and Breach, 1993.

[2] F.E. Benth. On the positivity of the stochastic heat equation. Potential Analysis, 6:127-148, 1997.

[3] F.E. Benth. On weighted $l^{2}(\omega)$-spaces, their duals and Itô integration. University of Aarhus, Preprint, MaPhySto 17, 1998.

[4] F.E. Benth, Th. Deck, and J. Potthoff. A white noise approach to a class of non-linear stochastic heat equations. J. Funct. Anal., 146:382-415, 1997.

[5] F.E. Benth and J. Gjerde. Convergence rates for finite element approximations of stochastic partial differential equations. Stochastics and Stochastic Reports, 63:313-326, 1998.

[6] F.E. Benth and H. Gjessing. A non-linear parabolic equation with noise. a reduction method, 1994.

[7] F.E. Benth and J. Potthoff. On the martingale property for generalized stochastic processes. Stochastics and Stochastic Reports, 58:349-367, 1996.

[8] T. Deck, J. Potthoff, and G: Våge. A review of white noise analysis from a probabilistic standpoint. Acta Appl. Math., 48(1):91-112, 1997.

[9] E.B Dynkin and A.A. Juschewitsch. Sätze und Aufgaben über Markoffsche Prozesse. Number 51 in Heidelberger Taschenbücher. Springer-Verlag, 1966.

[10] H.K. Gjessing. A variation of parameters solution of a quasilinear skorohod SDE using the Wick product. Stochastic analysis and related topics, VI (Geilo, 1996). Progr. Probab. 42, Birkhäuser Verlag, 1998.

[11] M. Grothaus. New Results in Gaussian Analysis and their Applications in Mathematical Physics. PhD thesis, University of Bielefeld, 1999.

[12] T. Hida, H.-H. Kuo, J. Potthoff, and L. Streit. White Noise - An Infinite Dimensional Calculus. Kluwer Academic Publishers, Dordrecht, 1993.

[13] H. Holden, B. Øksendal, J. Ubøe, and T.-S Zhang. Stochastic Partial Differential Equations - A Modeling, White Noise Functional Approach. Probability and its Applications. Birkhäuser, Boston, 1996.

[14] Y. Kondratiev, P. Leukert, and L. Streit. Wick calculus in Gaussian analysis. Acta Appl. Math., 44:269-294, 1996.

[15] Y. Kondratiev and L. Streit. Spaces of white noise distributions: constructions, descriptions, applications. Rep. Math. Phys., 33:341-366, 1993.

[16] H.-H. Kuo. White Noise Distributions. Probability and Stochastics Series. CRC Press, Baton Rouge, 1996.

[17] P. Malliavin. Stochastic Analysis. Number 313 in Grundlehren der mathematischen Wissenschaften. SpringerVerlag, 1997.

[18] J. Potthoff and M. Timpel. On a dual pair of smooth and generalized variables. Potential Analysis, 4:637-654, 1995.

[19] T.G. Theting. Solving wick-stochastic boundary value problems using a finite element method. Manuscript, 1999.

[20] G. Våge. Stochastic Differential Equations and Kondratiev Spaces. PhD thesis, Norwegian University of Technology and Science, 1995

[21] K. Yosida. Functional Analysis. Springer-Verlag, Berlin, 5 edition, 1978. 
(Fred Espen Benth)

Department of Mathematics

UNIVERSITY OF OSLO

P.O. BOX 1053, BLINDERN

N-0316 OSLO, NORWAY

AND

MaPhySto - Centre for Mathematical Physics and Stochastics

UNIVERSITY OF AARHUS

NY MUNKEgADE

DK-8000 Arhus, Denmark

E-mail address: fredbomath.uio.no

(Thomas Gorm Theting)

Department of Mathematics

NoRweglan UNIVERSITY OF SCIENCE aND TEChNOLOGY

N-7491 TRONDHEIM, NORWAY

AND

LEHRSTUHL FÜr. MATHEMATIK V

FAKULTÄT FÜR MATHEMATIK UND INFORMATIK

UNIVERSITÄT MANNHEIM

D-68131 Mannheim, Germany

E-mail address: tgt@math.ntnu.no 